\title{
MUSINGS
}

\section{Why have protein biomarkers not reached the clinic?}

\author{
Samir M Hanash*
}

It is disappointing that despite the substantial advances in our understanding of the molecular basis of disease, there is a paucity of approved biomarkers. Protein biomarkers in biological fluids in particular have the potential to inform regarding risk of disease or to allow early detection for more effective treatment. There is an equally appalling lack of other types of biomarkers, whether for disease classification for individualized therapy or for other applications. However, often the finger is pointed at protein biomarkers given the thousands of publications describing promising candidates. Few of these candidates have been pursued to support their clinical utility, and most of those that have been pursued have failed in subsequent validation studies. A case in point is a recent validation study encompassing 28 promising candidate protein biomarkers for ovarian cancer that were assayed in prediagnostic serum specimens from ovarian cancer cases and controls in the Prostate, Lung, Colorectal, and Ovarian Cancer Screening Trial. None of the individual markers exhibited performance characteristics that equaled those of the cancer antigen CA-125, the current best marker for ovarian cancer [1]. Neither did their combination into a panel outperform CA-125. The negative result of this study has been widely publicized as yet another piece of evidence that something is wrong in our approach to biomarkers.

\section{The paucity of biomarkers to reach the clinic transcends proteomics}

This doom and gloom view of the current state of biomarkers is only partly justified. Where do we place the blame and what can we do about it? First of all, in defense of proteomics, I would point out that the paucity of biomarkers to reach the clinic far transcends proteomics and protein-based biomarkers. The massive investment in genomics and transcriptomics, which far exceeds any

*Correspondence: shanash@fhcrc.org

Fred Hutchinson Cancer Research Center, 1100 Fairview Avenue, Seattle, WA 98109, USA investment in proteomics, has also yielded a very limited number of diagnostics with demonstrated clinical utility, despite the promise. For example, in 2008 the National Institutes of Health (NIH) funding for all proteomics across all NIH institutes was estimated at \$125 million [2], whereas in the same year funding for the National Human Genome Research Institute alone for its programs amounted to $\$ 487$ million [3]. It would seem that irrespective of the type of biomarkers being sought, whether DNA, RNA, protein or metabolite based, there is a fundamental problem in our approach to biomarkers.

The shortage of biomarkers is not for lack of trying, but may be attributed historically to insufficient resources and incentives to develop and maintain collaborative teams able to impact the problem. As a result, the effort is mostly single investigator based with limited means to accomplish objectives. This is reflected in publications of studies that generally consist of comparisons between a disease group of specimen(s) and a control group(s) leading to the discovery of biomarker candidates that seem promising but that need to be further pursued to determine their relevance. In retrospect, most such studies have not been solidly grounded in an understanding of the biological and molecular heterogeneity of disease states to guide the experimental design of biomarker discovery studies. Moreover, the technologies that have been utilized for discovery, particularly in the early days of proteomics, lacked sufficient depth to allow exploration of low abundance proteins and their modifications that represent a potentially rich source of biomarkers [4]. Even when all of the above limitations are met there has been a considerable shortage of quality specimens for discovery and validation studies that overcome the biases inherent in retrospective samples [5].

\section{The way out}

Clearly there is a need to reassess our strategies to discover and develop biomarkers. First we need to shed the notion that developing biomarkers is an easy process. It may be argued that developing biomarkers is as complex a process as developing therapeutics, necessitating a substantial investment with a high potential for failure. Consequently, such an investment can only be 


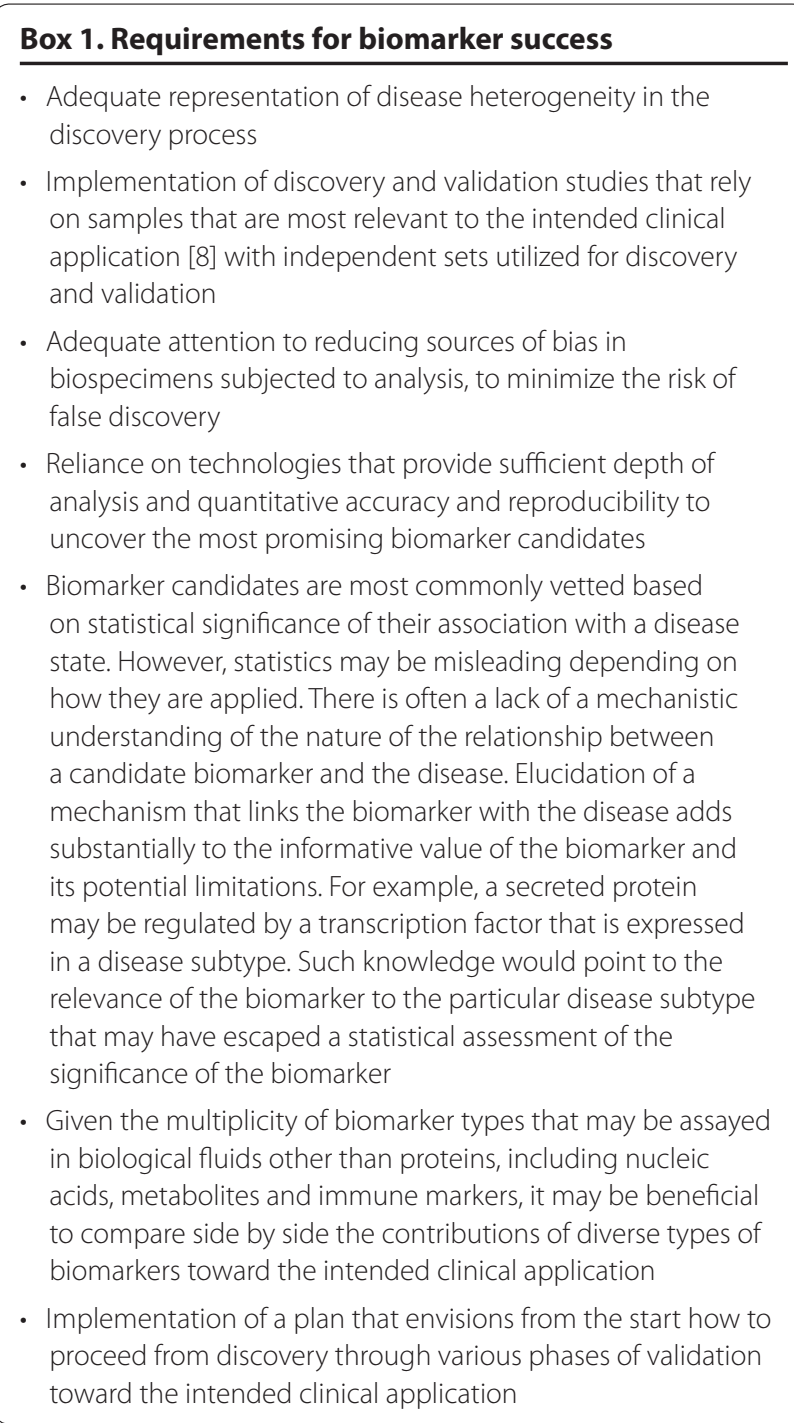

justified if the return on investment is substantial. At present, this is rarely the case, particularly for the protein type of biomarkers for which immunoassays are developed. The reimbursement for these types of biomarkers by insurance companies and governments is modest at best and consequently their development is not adequately incentivized. Most often, major diagnostics companies expect that candidate markers have reached a fairly advanced stage in their development and validation before expressing serious interest in their pursuit, resulting in a 'death valley' conundrum for biomarkers that are in the initial stages of their development but that do not elicit sufficient interest to be pursued further.

The challenge for the next decade is to implement road maps that fast track the development of biomarkers, whether protein, nucleic acid or metabolite based, to reach the clinic in an efficient manner. It will 'take a village' to implement a paradigm shift in our approach to biomarkers, necessitating a partnership among the various stakeholders, from academia with multidisciplinary contributions, to philanthropy, government and industry. Critical to this effort is the need to address some key requirements, as outlined in Box 1.

Initiatives aimed at contributing to a paradigm shift in our approach to the development of biomarkers are burgeoning. They are exemplified by the increased availability of biospecimens through cohort studies with reduced bias. They are also exemplified by consortia to assess and standardize technologies for discovery and assays of biomarker candidates - for example, privatepublic partnerships to develop biomarkers for particular diseases as in the case of the Canary Foundation [6] and the National Cancer Institute Early Detection Research Network partnership to develop early detection biomarkers for lung and prostate cancer. Another noteworthy initiative is that of the international WIN Consortium [7], which plans to launch a range of research and clinical projects to improve early diagnosis of cancer and to establish new treatment strategies that encompass companion diagnostics.

Hopefully, an appreciation of the above considerations would alter our current perceptions regarding biomarkers in general and protein biomarkers in particular. Addressing the key requirements for success in this field by the stakeholders would help ensure that we are no longer faced with a paucity of approved biomarkers.

\section{Abbreviations}

$\mathrm{NIH}$, National Institutes of Health.

\section{Competing interests}

The author declares that he has no competing interests.

\section{Published: 26 October 2011}

\section{References}

1. Mai PL, Wentzensen N, Greene MH: Challenges related to developing serum-based biomarkers for early ovarian cancer detection. Cancer Prev Res (Phila) 2011, 4:303-306.

2. Fong T: NIH Proteomics Funding Fell 3 Percent in FY 2008 [http://2429-genomeweb.voxcdn.com/proteomics/ nih-proteomics-funding-fell-3-percent-fy-2008]

3. AAAS: NIH Budgets by Institute and Funding Mechanism, FY 1998-2008 [http://www.aaas.org/spp/rd/health08c.pdf]

4. Hanash S, Taguchi A: The grand challenges of cancer proteomics. Nat Rev Cancer 2010, 10:652-660.

5. McClave CB: Biospecimens: Advancing Epidemiologic Eesearch [http://dceg.cancer.gov/newsletter/jul09/0709_biospecimens.shtml]

6. Canary Foundation [http://www.Canaryfoundation.org]

7. WIN Consortium: Worldwide Innovative Networking in Personalized Cancer Medicine [http://www.winconsortium.org]

8. Pepe MS, Feng Z, Janes H, Bossuyt PM, Potter JD: Pivotal evaluation of the accuracy of a biomarker used for classification or prediction: standards for study design. J Natl Cancer Inst 2008, 100:1432-1438.

doi:10.1186/gm282

Cite this article as: Hanash SM: Why have protein biomarkers not reached the clinic? Genome Medicine 2011, 3:66. 